\title{
Internal Limiting Membrane Peeling and Flap Inverting under Air in Large Idiopathic Macular Hole Surgery
}

\author{
Yuan Zong $\mathbb{D}^{1,2}$ Kaicheng Wu, ${ }^{1,2}$ Jian Yu $\mathbb{D}^{1,2}$ Changbo Zhou $\mathbb{D}^{3},{ }^{3}$ and Chunhui Jiang $\mathbb{D}^{1,2}$ \\ ${ }^{1}$ Department of Ophthalmology and Vision Science, Eye and ENT Hospital, Fudan University, Shanghai 200031, China \\ ${ }^{2}$ Key Laboratory of Myopia of State Health Ministry, Key Laboratory of Visual Impairment and Restoration of Shanghai, \\ Shanghai 200031, China \\ ${ }^{3}$ Department of Ophthalmology, Zhejiang Putuo Hospital, Zhoushan, Zhejiang Province, China
}

Correspondence should be addressed to Changbo Zhou; drzcb@qq.com and Chunhui Jiang; chhjiang70@163.com

Received 16 April 2021; Revised 16 August 2021; Accepted 8 September 2021; Published 25 September 2021

Academic Editor: Manuel S. Falcão

Copyright (C) 2021 Yuan Zong et al. This is an open access article distributed under the Creative Commons Attribution License, which permits unrestricted use, distribution, and reproduction in any medium, provided the original work is properly cited.

\begin{abstract}
Purpose. To evaluate the efficacy of internal limiting membrane (ILM) peeling combined with modified flap inverting under air in the treatment of large idiopathic macular hole $(\mathrm{MH})$. Methods. Eyes with a large idiopathic $\mathrm{MH}$ (minimum diameter $>550 \mu \mathrm{m}$ ) were included in this study. The surgical procedure included standard 23-gauge pars plana vitrectomy (PPV), ILM peeling, complete fluid-gas exchange, and ILM flap inversion under air. The patients underwent follow-up exam including optical coherence tomography (OCT) and best-corrected visual acuity (BCVA) measurement. Results. Sixteen eyes from 16 patients were included. Mean MH diameter was $681.43 \pm 112.12 \mu \mathrm{m}$. After a mean follow-up time of $6.25 \pm 2.65$ months, in all cases, the MH was closed, and the ILM flap could be seen at the inner surface of the fovea. U-shaped and V-shaped MH closure was achieved in 11 and 5 cases, respectively. The BCVA improved significantly from $1.49 \pm 0.35 \log$ MAR to $0.89 \pm 0.35 \log$ MAR $(p<0.05)$, and visual acuity of 20/100 or better was achieved in 8 eyes. Conclusion. ILM flap inverting under air was helpful in improving the functional and anatomic outcomes of vitrectomy for large idiopathic $\mathrm{MH}$.
\end{abstract}

\section{Introduction}

Idiopathic macular hole $(\mathrm{MH})$ is a common finding in the clinic, with an incidence of about 8.5 individuals per 100,000 population per year [1]. In 1991, Kelly and Wendel [2] first reported the treatment of $\mathrm{MH}$ using pars plana vitrectomy $(\mathrm{PPV})$. Although the primary result was encouraging, $2 \%-$ $32 \%$ of the $\mathrm{MH}$ closure cases remained unclosed [2-4]. Many other methods have been tried to improve the outcome [5, 6]. In 2010, Michalewska et al. [7] introduced the inverted internal limiting membrane (ILM) flap technique, which greatly improved the postoperative closure rate for large $\mathrm{MH}$ and has been widely used since then. This technique has certain drawbacks such as easy detachment of the ILM flap from the edge of the MH during fluid-air exchange [8]. Many researchers have devoted their time to modify this technique to overcome these limitations $[9,10]$. Herein, we describe a modified method, manipulating the ILM flap under air; furthermore, the surgical outcomes of this modified technique were evaluated.

\section{Methods}

This retrospective study was approved by the Ethics Board of the Eye, Ear, Nose, and Throat (EENT) Hospital, Fudan University, and the procedures adhered to the tenets of the Declaration of Helsinki. All participants had given their written informed consent. Patients who were diagnosed with a large idiopathic $\mathrm{MH}$ with a diameter of $>550 \mu \mathrm{m}$ [11] and treated by a single surgeon $(\mathrm{CH} . \mathrm{J})$ at the EENT Hospital between April 2019 and January 2020 were recruited. The inclusion criteria were as follows: clinical presentation of $\mathrm{MH}$ with a diameter of $>550 \mu \mathrm{m}$, without retinal detachment; intraocular pressure of $<21 \mathrm{mmHg}$, axial length (AL) between 21 and $25 \mathrm{~mm}$, and SE between +1 and -3 diopter (D). Patients with a history of proliferative 
vitreoretinopathy, intraocular diseases other than cataract, and those who underwent retinal surgery previously were excluded. Data of the general medical history and ophthalmic history were carefully recorded. Standard eye examinations were performed before surgery and at each visit after surgery, including best-corrected visual acuity (BCVA), slit-lamp biomicroscopy examination, and dilated fundus examination using a slit-lamp with an $84 \mathrm{D}$ lens. Axial length (AL) was measured before surgery using an optical biometer (IOLMaster, version 3.01; Carl Zeiss Meditec, Jena, Germany), and intraocular pressure (IOP) was determined using a noncontact tonometer (Canon TX-20 full auto tonometer, Canon, Japan).

Intraoperative and postoperative complications were recorded. Optical coherence tomography (OCT) (using SPECTRALIS HRA OCT; Heidelberg Engineering, Heidelberg, Germany) was performed before and at 1 week and $1,3,6$, and 9 months after surgery. $\mathrm{MH}$ diameter was defined as the minimum opening diameter as measured on horizontal OCT scans across the center [12]. Macular closure type was defined according to the classification detailed by Imai et al. [13]: $U$-shaped closure is similar to that of the normal macula that has a smooth circular surface. V-shaped closure is a steep foveal contour that has a notch where the neurosensory layer is extremely thin. W-shaped closure has the foveal defect of the neurosensory retina with flattened cuff.

2.1. Surgical Technique. All surgeries were performed by a single retina specialist $(\mathrm{CH}$. J) under a retrobulbar block. The pupil was dilated with a combination of phenylephrine 5\% and tropicamide $1 \%$. The cataract was removed, and the posterior capsule was left intact. Then, standard 3-port 23gauge PPV was performed with a noncontact wide-angle viewing system. The Resight 500 was used in 14 cases, and the Oculus BIOM $^{\circledR} 5$ system was used in 2 cases. Vitrectomy was first performed, followed by posterior hyaloid removal with the help of triamcinolone acetonide. After that, the ILM was stained with indocyanine green (ICG, $2.5 \mathrm{mg} / \mathrm{mL}$, Dandong Yichang Pharmaceutical Co., China), and the ILM was peeled starting from the inferior vascular arcade. The peeling was stopped at a distance about $50-100 \mu \mathrm{m}$ from the edge of the MH. After the peeling of the $360^{\circ}$ ILM, the ILM flap was trimmed using a vitreous cutter, and the central part (about 1.5 papillary diameter) was left attached to the edge of the hole. Then, complete fluid-gas exchange was performed, and the air pressure lowered to about $20 \mathrm{mmHg}$. The ILM flap was then inverted. Several attempts were occasionally required before the flap was picked and inverted (ILM flap inverted step under air in two cases, https://drive.google.com/file/d/ 1MEKTMQQM4J_TAYAr7iA51f6Oi_mm59rJ/view?usp=sh aring). The reflex from the interface between air and fluid would change when the ILM forceps reached the interface; sometimes, this could be used as a sign for the moment to pick the ILM flap. The ILM flap could be inverted over the $\mathrm{MH}$ in two or more directions. If there was too much balanced salt solution (BSS) over the posterior pole, then the BSS was removed using a backflush needle. And in case, the flap moved from the ideal place with the removal of BSS, the procedure of ILM inverting was performed again. The trocars were then removed. Patients were introduced to have a facedown positioning for 7 days postoperatively.

2.2. Statistics. All analyses were performed using SPSS software, version 16.0 (SPSS Inc., Chicago, USA). The level of significance was set at $p<0.05$. Data are presented as the mean \pm standard deviation (SD). Student's $t$-test was used to compare the preoperation and postoperation data.

\section{Results}

Sixteen eyes from 16 patients with a large idiopathic $\mathrm{MH}$ were included in this study. Patients' demographic data are given in Table 1. Mean symptom duration was $29.06 \pm 23.24$ months, and mean AL was $23.17 \pm 0.84 \mathrm{~mm}$. The mean diameter of the MH was $681.44 \pm 112.12 \mu \mathrm{m}$. In case 5 , a very tiny retinal hemorrhage was noticed at the place where the ILM was picked. No other intraoperative or postoperative complications were noted.

In 15 cases, the $\mathrm{MH}$ closure was achieved after primary surgery (Figures 1(a)-1(f)). While in case 2, with the ILM over the MH (Figure $1(\mathrm{~g})$ ), the latter remained open after the first operation. The patient received a second fluid-air exchange and ILM manipulation under air. A U-shaped closure was achieved 3 months later (Figure $1(\mathrm{~h})$ ). After a mean follow-up duration of $6.25 \pm 2.65$ months, the BCVA improved significantly from $1.49 \pm 0.35 \log$ MAR to $0.89 \pm 0.35$ logMAR $(p<0.05)$; visual acuity of $20 / 100$ or better was achieved in 8 eyes. In all cases, the MH was closed, and the ILM could be seen at the inner surface of the retina at the fovea. In 11 cases, a U-shaped MH closure was achieved, and in the other 5 cases, a V-shaped MH closure was achieved (Figure 1).

\section{Discussion}

Using a modified ILM flap inverting technique, large idiopathic MHs were successfully treated with PPV and air tamponade. Postsurgery OCT examination confirmed that, in all cases, the MH was closed and the ILM was lying over the central fovea.

With PPV, ILM peeling, and gas tamponade, most of the idiopathic MH cases could be successfully treated. For refractory cases, ILM flap inverting has been developed, but always with expansible gas or silicone and face down position. Even so, closure rate was not $100 \%$ [14]. Michalewska et al. [7] suggested the inverted ILM could be regarded as a scaffold for tissue proliferation and may provide an environment in which photoreceptors can assume new positions in direct proximity to the fovea. Also, the ILM contains Müller cell fragments. However, in some cases, MH closure was still not achieved, possibly because the ILM flap detached spontaneously after the fluid-air exchange [7]. To solve this problem, here we reported a modification of ILM peeling and flap inverting technique-invert ILM flap under air. During follow-up, in all cases, MH closure was achieved 
TABLE 1: Demographics of patients with a large macular hole.

\begin{tabular}{|c|c|c|c|c|c|c|c|c|c|c|c|}
\hline \multirow[t]{2}{*}{ Patient no. } & \multirow[t]{2}{*}{ Age (years) } & \multirow[t]{2}{*}{ Gender } & \multirow[t]{2}{*}{ Laterality } & \multirow[t]{2}{*}{$\mathrm{AL}(\mathrm{mm})$} & \multirow{2}{*}{$\begin{array}{c}\mathrm{MH} \\
\text { diameter }(\mu \mathrm{m})\end{array}$} & \multicolumn{2}{|c|}{ BCVA (Snellen) } & \multicolumn{2}{|c|}{$\begin{array}{c}\text { BCVA } \\
(\log M A R)\end{array}$} & \multirow[t]{2}{*}{ Follow-up (months) } & \multirow{2}{*}{$\begin{array}{c}\text { Type of } \\
\text { hole } \\
\text { closure }\end{array}$} \\
\hline & & & & & & Initial & Final & Initial & Final & & \\
\hline 1 & 64 & $\mathrm{~F}$ & Right & 23.10 & 618 & $20 / 200$ & $20 / 100$ & 1.0 & 0.7 & 7 & $\mathrm{U}$ \\
\hline 2 & 72 & $\mathrm{~F}$ & Right & 23.64 & 600 & $20 / 400$ & $20 / 100$ & 1.3 & 0.7 & 3 & $\mathrm{U}$ \\
\hline 3 & 59 & $\mathrm{~F}$ & Right & 23.29 & 603 & $20 / 1000$ & $20 / 200$ & 1.7 & 1.0 & 5 & $\mathrm{~V}$ \\
\hline 4 & 65 & $\mathrm{~F}$ & Right & 23.69 & 696 & $20 / 2000$ & $20 / 133$ & 2.0 & 0.8 & 8 & $\mathrm{~V}$ \\
\hline 5 & 76 & $\mathrm{~F}$ & Left & 22.08 & 914 & $20 / 1000$ & $20 / 1000$ & 1.7 & 1.7 & 8 & $\mathrm{~V}$ \\
\hline 6 & 67 & $\mathrm{~F}$ & Left & 22.32 & 609 & $20 / 1000$ & $20 / 400$ & 1.7 & 1.3 & 9 & $\mathrm{U}$ \\
\hline 7 & 68 & $\mathrm{~F}$ & Right & 22.30 & 669 & $20 / 1000$ & $20 / 100$ & 1.7 & 0.7 & 10 & $\mathrm{~V}$ \\
\hline 8 & 65 & $\mathrm{~F}$ & Right & 21.96 & 574 & $20 / 200$ & $20 / 50$ & 1.0 & 0.4 & 4 & $\mathrm{U}$ \\
\hline 9 & 69 & M & Left & 22.90 & 627 & $20 / 200$ & $20 / 67$ & 1.0 & 0.52 & 10 & $\mathrm{U}$ \\
\hline 10 & 49 & $\mathrm{~F}$ & Right & 22.54 & 920 & $20 / 400$ & $20 / 200$ & 1.3 & 1.0 & 4 & $\mathrm{U}$ \\
\hline 11 & 69 & $\mathrm{~F}$ & Left & 23.33 & 716 & $20 / 400$ & $20 / 200$ & 1.3 & 1.0 & 3 & $\mathrm{U}$ \\
\hline 12 & 67 & M & Right & 23.67 & 550 & $20 / 667$ & $20 / 100$ & 1.5 & 0.7 & 10 & $\mathrm{U}$ \\
\hline 13 & 71 & $\mathrm{~F}$ & Right & 24.06 & 626 & $20 / 400$ & $20 / 67$ & 1.3 & 0.51 & 6 & $\mathrm{U}$ \\
\hline 14 & 48 & $\mathrm{~F}$ & Right & 24.56 & 796 & $20 / 2000$ & $20 / 333$ & 2 & 1.22 & 6 & $\mathrm{~V}$ \\
\hline 15 & 70 & $\mathrm{~F}$ & Left & 22.67 & 746 & $20 / 400$ & $20 / 100$ & 1.3 & 0.7 & 3 & $\mathrm{U}$ \\
\hline 16 & 57 & $\mathrm{~F}$ & Left & 24.67 & 639 & $20 / 2000$ & $20 / 400$ & 2 & 1.3 & 4 & $\mathrm{U}$ \\
\hline
\end{tabular}

$\mathrm{AL}$, axial length; $\mathrm{MH}$, macular hole; BCVA, best-corrected visual acuity.
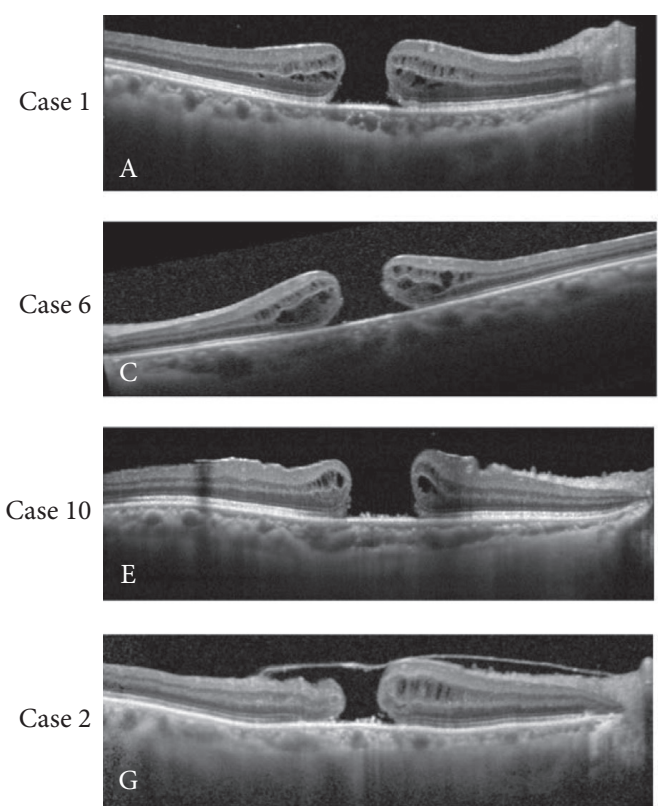
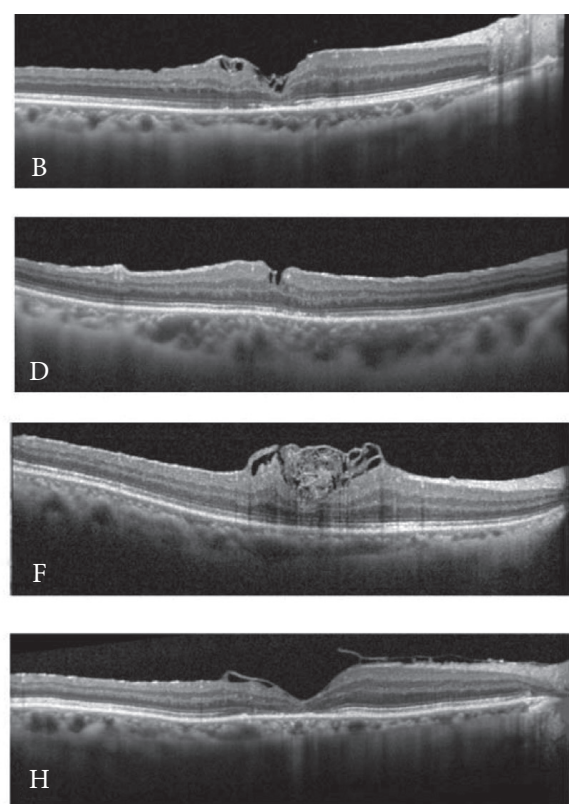

FIgURE 1: Preoperative and postoperative optical coherence tomographic (OCT) scan images of four cases. (a-b) Preoperative and postoperative OCT scan images of case 1. (c-d) Scan images of case 6. (e-f) Scan images of case 10. (g-h) Scan images of case 2 before and after the second procedure.

and the ILM flap could be seen at the inner surface of the fovea.

On the other hand, for special MHs, including large, persistent, and highly myopic, special techniques were developed such as perfluorocarbon liquid-assisted inverted limiting membrane flap technique [15], internal limiting membrane transposition and tuck technique [16], inverted ILM flap combined autologous blood clot technique [17], the neurosensory retinal flap [18], human amniotic membrane plug transplant $[19,20]$, and lens capsular flap transplantation [21]. These techniques achieved a high success rate, but limitations such as retinal toxicity and risk of infection were also noticed (Supplementary Table). The modification we presented this time has certain advantages. First, one can be sure about the position of the ILM flap lying over the $\mathrm{MH}$ at the end of the surgery. During follow-up visits, the ILM was found above the fovea in all the cases. In case 2, although the MH remained open, the ILM was seen over the MH. It is worth mentioning that although there are several ILM layers seen in case 11 postoperation, the inverted 
ILM flap technique was used in our study which was different from ILM insertion. The neurosensory retina showed a complete recovery in case 11 . While in former studies, ILM insertion or inverted over the hole had different outcomes on outer nuclear layers, even if the hole closes [22].

Second, this modification is simple, straightforward, and does not require any additional procedure, instrument, or material, making it more economical and greatly reducing the risk of infection and retinal toxicity. Furthermore, in cases where the hole persists, one can perform fluid-air exchange and repeat the procedure again, as we detailed in case 2. Moreover, during the study period, long-term gas was not available in China; hence, only air was used in our cases. Thus, with this modification, the prone positioning time, which was required after $\mathrm{MH}$ surgery, could be reduced remarkably. Also, the large macular hole closed in all cases. Compared to other studies $[11,14]$, our patients had a longer average duration of disease (average 29.06 \pm 23.24 months) and the baseline vision was poor $(1.49 \pm 0.35 \operatorname{logMAR})$; however, they had a mean 0.6 logMAR improvement in BCVA, which was comparable to or even better than that reported in other studies (Supplementary Table).

Some key points should be noted about the procedure. The first point is to provide a clear view of the posterior pole. The posterior capsule must be left intact and kept moist. With a dried posterior capsule or posterior IOL surface, the view would be extremely poor and manipulation of the ILM flap under air would be near-impossible. In all our cases, the wide-angle viewing system was used, and the author found it difficult to perform this technique with a flat contact lens. The second point is the control of BSS over the posterior pole. The reflex from the interface between air and fluid would change when the ILM forceps touches the interface. This could be a sign for when to pick the ILM flap with the ILM forceps. With too much BSS, this sign does not work well. With excess BSS, the ILM might not stay stable at the place it was left. Using a backflush needle, the extra BSS should be removed. Usually, after a thorough fluid-air exchange, within a few seconds, some BSS would be accumulated over the area, and this would be the optimal moment to pick the ILM flap. Furthermore, as only a very tiny part of the ILM was left connected to the retina underneath, the air pressure was lowered to around $20 \mathrm{mmHg}$ when the remaining fluid at the posterior pole was cleaned. With high air pressure, the ILM flap might accidentally disappear into the backflush needle with the fluid. One might notice the hemorrhage at the macular area in https://drive.google.com/file/d/1MEKTMQQM4J_TAY Ar7iA51f6Oi_mm59rJ/view?usp=sharing, and this was a result of the low intraocular pressure. Iatrogenic damage to the retina might be of concern; in only one of our initial cases, a very tiny retinal hemorrhage was noticed at the place the ILM was picked. In this study, we presented the results of our primary cases, and we have used this modification in more than 20 cases; no other intra or postoperative complications were noticed.

However, our cases were limited, and we tried this surgery in highly myopic cases, but the posterior staphyloma made the visualization more challenging; the efficacy of this surgery in highly myopic cases or other macular surgery needs to be studied in the future.

With our primary results, ILM flap inverting under air was helpful in improving the functional and anatomic outcomes of vitrectomy for large idiopathic $\mathrm{MH}$.

\section{Data Availability}

The data used to support the findings of this study are available from the corresponding author upon request.

\section{Ethical Approval}

The study was approved by the Institutional Review Board of the Eye and ENT Hospital of Fudan University, Shanghai, China (No. 2019031-1).

\section{Consent}

All subjects had given their written informed consent.

\section{Conflicts of Interest}

The authors declare that they have no conflicts of interest.

\section{Authors' Contributions}

Yuan Zong and Kaicheng Wu contributed equally to this work.

\section{Acknowledgments}

This study was supported by research grants from the National Key R\&D Program of China (2017YFC0108200) and the Shanghai Committee of Science and Technology (19441900900 and 201409006800).

\section{Supplementary Materials}

Supplementary Table. Comparison of special techniques used for refractory macular holes. (Supplementary Materials)

\section{References}

[1] C. A. McCannel, J. L. Ensminger, N. N. Diehl, and D. N. Hodge, "Population-based incidence of macular holes," Ophthalmology, vol. 116, no. 7, pp. 1366-1369, 2009.

[2] N. E. Kelly and R. T. Wendel, "Vitreous surgery for idiopathic macular holes," Archives of Ophthalmology, vol. 109, no. 5, pp. 654-659, 1991.

[3] C. Haritoglou, A. Gandorfer, A. Kampik, and D. Tognetto, "Anatomic and visual outcomes after indocyanine greenassisted peeling of the retinal internal limiting membrane in idiopathic macular hole surgery," American Journal of Ophthalmology, vol. 138, no. 4, pp. 691-692, 2004.

[4] J. Beutel, G. Dahmen, A. Ziegler, and H. Hoerauf, "Internal limiting membrane peeling with indocyanine green or trypan blue in macular hole surgery," Archives of Ophthalmology, vol. 125, no. 3, pp. 326-332, 2007.

[5] J. T. Thompson, B. M. Glaser, R. N. Sjaarda, R. P. Murphy, and A. Hanham, "Effects of intraocular bubble duration in the 
treatment of macular holes by vitrectomy and transforming growth factor-beta 2," Ophthalmology, vol. 101, no. 7, pp. 1195-1200, 1994.

[6] T. W. Olsen, P. Sternberg Jr., A. Capone Jr., et al., "Macular hole surgery using thrombin-activated fibrinogen and selective removal of the internal limiting membrane," Retina, vol. 18, no. 4, pp. 322-329, 1998.

[7] Z. Michalewska, J. Michalewski, R. A. Adelman, and J. Nawrocki, "Inverted internal limiting membrane flap technique for large macular holes," Ophthalmology, vol. 117, no. 10, pp. 2018-2025, 2010.

[8] I. Chatziralli, G. Machairoudia, D. Kazantzis, G. Theodossiadis, and P. Theodossiadis, "Inverted internal limiting membrane flap technique for myopic macular hole: a meta-analysis," Survey of Ophthalmology, vol. 66, no. 5, pp. 771-780, 2021.

[9] Y. Hirata, K. Yuda, D. Odontuya, T. Hayashi, and Y. Suzuki, "A viscoelastic aspiration technique for autologous transplantation of the free-flap inner limiting membrane during macular hole surgery," Retina, vol. 39, no. 1, pp. S87-S91, 2019.

[10] M. K. Shin, K. H. Park, S. W. Park, I. S. Byon, and J. E. Lee, "Perfluoro-n-octane-assisted single-layered inverted internal limiting membrane flap technique for macular hole surgery," Retina, vol. 34, no. 9, pp. 1905-1910, 2014.

[11] T. Yamashita, T. Sakamoto, H. Terasaki et al., "Best surgical technique and outcomes for large macular holes: retrospective multicentre study in Japan," Acta Ophthalmologica, vol. 96, no. 8, pp. e904-e910, 2018.

[12] S. Ullrich, C. Haritoglou, C. Gass, M. Schaumberger, M. W. Ulbig, and A. Kampik, "Macular hole size as a prognostic factor in macular hole surgery," British Journal of Ophthalmology, vol. 86, no. 4, pp. 390-393, 2002.

[13] M. Imai, H. Iijima, T. Gotoh, and S. Tsukahara, "Optical coherence tomography of successfully repaired idiopathic macular holes," American Journal of Ophthalmology, vol. 128, no. 5, pp. 621-627, 1999.

[14] J. H. Park, S. M. Lee, S. W. Park, J. E. Lee, and I. S. Byon, "Comparative analysis of large macular hole surgery using an internal limiting membrane insertion versus inverted flap technique," British Journal of Ophthalmology, vol. 103, no. 2, pp. 245-250, 2019.

[15] Z. Hu, X. Gu, H. Qian et al., "Perfluorocarbon liquid-assisted inverted limiting membrane flap technique combined with subretinal fluid drainage for macular hole retinal detachment in highly myopic eyes," Retina, 2019.

[16] N. S. K. Fung, A. K. H. Mak, R. Yiu, I. Y. H. Wong, and W. C. Lam, "Treatment of large, chronic and persistent macular hole with internal limiting membrane transposition and tuck technique," International Journal of Retina and Vitreous, vol. 6, no. 1, p. 3, 2020.

[17] Z. Hu, H. Lin, Q. Liang, and R. Wu, "Comparing the inverted internal limiting membrane flap with autologous blood technique to internal limiting membrane insertion for the repair of refractory macular hole," International Ophthalmology, vol. 40, no. 1, pp. 141-149, 2020.

[18] D. S. Grewal and T. H. Mahmoud, "Autologous neurosensory retinal free flap for closure of refractory myopic macular holes," JAMA Ophthalmology, vol. 134, no. 2, pp. 229-230, 2016.

[19] S. Rizzo, T. Caporossi, R. Tartaro et al., "A human amniotic membrane plug to promote retinal breaks repair and recurrent macular hole closure," Retina, vol. 39, no. 1, pp. S95-S103, 2019.
[20] M. A. Ferreira, A. Maia, A. J. Machado et al., "Human amniotic membrane for the treatment of large and refractory macular holes: a retrospective, multicentric, interventional study," International Journal of Retina and Vitreous, vol. 7, no. 1, p. 38, 2021.

[21] S.-N. Chen and C.-M. Yang, "Lens capsular flap transplantation in the management of refractory macular hole from multiple etiologies," Retina, vol. 36, no. 1, pp. 163-170, 2016.

[22] M. Y. Faria, H. Proença, N. G. Ferreira, D. C. Sousa, E. Neto, and C. Marques-Neves, "Inverted internal limiting membrane flap techniques and outer retinal layer structures," Retina, vol. 40, no. 7, pp. 1299-1305, 2020. 